\title{
Local versus field scale soil heterogeneity characterization - a challenge for representative sampling in pollution studies
}

\author{
Z. Kardanpour ${ }^{1,2}$, O. S. Jacobsen ${ }^{2}$, and K. H. Esbensen ${ }^{1,2}$ \\ ${ }^{1}$ Geological Survey of Denmark and Greenland (GEUS), Copenhagen, Denmark \\ ${ }^{2}$ ACABS research group, University of Aalborg, campus Esbjerg (AAUE), Esbjerg, Denmark
}

Correspondence to: Z. Kardanpour (zka.geus@gmail.com)

Received: 12 May 2015 - Published in SOIL Discuss.: 9 June 2015

Revised: 17 October 2015 - Accepted: 9 November 2015 - Published: 10 December 2015

\begin{abstract}
This study is a contribution to development of a heterogeneity characterization facility for "nextgeneration" soil sampling aimed, for example, at more realistic and controllable pesticide variability in laboratory pots in experimental environmental contaminant assessment. The role of soil heterogeneity in quantification of a set of exemplar parameters is described, including a brief background on how heterogeneity affects sampling/monitoring procedures in environmental pollutant studies. The theory of sampling (TOS) and variographic analysis has been applied to develop a more general fit-for-purpose soil heterogeneity characterization approach. All parameters were assessed in large-scale transect $(1-100 \mathrm{~m})$ vs. small-scale $(0.1-0.5 \mathrm{~m})$ replication sampling point variability. Variographic profiles of experimental analytical results from a specific well-mixed soil type show that it is essential to sample at locations with less than a $2.5 \mathrm{~m}$ distance interval to benefit from spatial auto-correlation and thereby avoid unnecessary, inflated compositional variation in experimental pots; this range is an inherent characteristic of the soil heterogeneity and will differ among other soils types. This study has a significant carrying-over potential for related research areas, e.g. soil science, contamination studies, and environmental monitoring and environmental chemistry.
\end{abstract}

\section{Introduction}

All parameters for realistic, effective integration of variability over different scales are directly related to soil heterogeneity. There is a growing need for an integrated understanding of contaminant behaviour in soil pollution studies (Arias-Estévez et al., 2008; Crespin et al., 2001; Johnsen et al., 2013; Li et al., 2006; Rodriguez-Cruzet al., 2006; Sørensen et al., 2006; Torstensson and Stark 1975; Rasmussen et al., 2005). In this context there is a missing link in the form of soil heterogeneity and its effective characterization, a feature often overlooked. Heterogeneity characterization is the first, and in some cases the most important, step in soil contaminant studies, with relationships to various other aspects of environmental research and monitoring. A result of introducing more valid soil heterogeneity characterization will be improved soil sampling procedures (Kardanpour et al., 2014, 2015a, b), which in turn will contribute towards improved environmental fate study reliability (Boudreault et al., 2012; Chappell and Viscarra Rossel, 2013; De Zorzi et al., 2008; Lin et al., 2013; Mulder et al., 2013; Totaro et al., 2013).

Even in simple systems, the variability and risk of misinterpretation may have a strong effect on parameterization of processes relating to compound fate studies. These latter issues are being increasingly more recognized, as is the lack of appropriate methods to ensure documented representativity of the experimental batch volumes/masses with respect to the surrounding geology and biotic/abiotic soil characteristics. There is an urgent need for scientifically based experimental approaches, scale-up procedures, and attendant principles for parameterization of variability in these types of natural systems (Kardanpour et al., 2014; Adamchuk et al., 2011; Chappell and Viscarra Rossel, 2013; De Zorzi et al., 2008). 
Of particular interest will be a newly developed facility for empirical variability characterization, which allows heterogeneity to be mapped at problem-dependent scale hierarchies. Based on this, it is possible to devise optimized sampling strategies that will allow fit-for-purpose representativity with respect to laboratory experiments depending on similar (or at least comparable) soil samples (pots). For this purpose, the theory of sampling (TOS) delivers benchmark measures expressing acceptable maximum heterogeneity limits and, in the case of violations/transgressions, furthers a complete understanding of how to identify and eliminate the detrimental sampling errors, as well as providing tools for unambiguous mixing effectiveness. By combining these tools with specific knowledge on the relevant contaminant processes and compound properties, it will be possible to address the critical scale-dependent variability with increased confidence based on more realistic environmental parameter delineation.

We here introduce the variographic approach mainly for the cases of 1-D as a means of characterizing the heterogeneity in one transect direction. Compared to the typical major variability in the $Z$ direction of soil depth profiles (soil horizons, layers, and geological formations), the linear (1D) or 2-D heterogeneity within soil horizons is significantly smaller, although this is exactly the kind of heterogeneity the present study aims at controlling. Contrary to depth profile zonation, among other things, the within-horizon 1-D and 2$D$ heterogeneity complies with the requirements of both TOS and geostatistics - i.e. spatial heterogeneity can be modelled variographically with regard to a physically meaningful $a v-$ erage level (the inherent stationarity assumption in geostatistics). It is not meaningful to apply variographic characterization on measurement series which contain discontinuous shifts, oversets, or other disrupting level changes, as is the prime characterization of soil depth zonations. The geostatistical tradition of modelling 2-D patterns based on projection onto a 1-D transect is also not free from debatable issues. The present authors do not wish to reject the 2-D geostatistical tradition with this statement, but in relation to the present matters this issue is better deferred to another occasion in which the 2-D modelling issue can be presented and discussed in full - this issue is a legitimate and interesting area for a fruitful debate. Entering into a 3-D geostatistical modelling realm, there are also here issues that in need of further discussion, e.g. the required minimum number of samples (measurements) needed for meaningful and stable variogram calculation. The present foray only aims at presenting the power of a simple 1-D variogram characterization operator based on TOS, upon which several versions of potential follow-up generalizations to 2-D and 3-D cases may be entertained. In the present context all isotropic 2-D heterogeneity patterns can be characterized comprehensively by a randomly selected 1-D direction (transect). In all sampling operations there should preferentially always be some sort of random selection involved, unless compelling geoscientific reasons exist for choosing a direction related to the genesis of the specific heterogeneity are met with, e.g. choosing a 1-D transect along a dominant plow direction.

This study focuses on development of the necessary heterogeneity characterization for sampling/monitoring and multi-parameter modelling practices, allowing implementation of realistic pesticide variability in experimental environmental contaminant assessment studies. The study has a significant carrying-over potential for related research areas, e.g. soil science, contamination studies, and environmental monitoring.

We here focus on characterization of soil heterogeneity in terms of soil moisture, organic matter (loss on ignition, LOI), biomass, microbiology, MCPA sorption, and mineralization. The measured parameters are here used to illustrate effective management of heterogeneity; this particular location has been studied before in its own right. Following two earlier complementary studies, the focus below is on the necessary representativity demands when facing compound fate and mineralization studies (Kardanpour et al., 2014, 2015). Field observation indicates a very well mixed sandy soil with almost no visual heterogeneity features. But the main issue is, does this apparent uniformity extend to all fate compounds? How is it possible to document that small sample masses, as typically used in pot experiments, are representative of their entire parent field, or to which sub-field scale? In other words, how can results and conclusions from laboratory experiments be reliably scaled up and generalized to larger scales?

\section{Materials and methods}

\subsection{Location and sampling pattern}

Fladerne Bæk is situated on the Karup periglacial outwash plain, Jutland, Denmark ( $\left.56^{\circ} \mathrm{N}, 9^{\circ} \mathrm{E}\right)$, south-west of Karup airport. The substratum is an arable sandy soil which has been tilled and cropped for more than 100 years, mainly supporting barley and potatoes during last 30 years. Thus this is a typical "very well mixed" soil type compared to the much more heterogeneous glacial clayey soil types treated in Kardanpour et al. (2014). Soil samples were collected from the topsoil (A horizon) in cylindrical cores; the present samples cover the depth interval from 0 to $15 \mathrm{~cm}$. The $60 \mathrm{~m}$ long sampling transect ran roughly north-south. Each field sample included 200-300 g of fresh soil. At the centre of this transect at point 29 , seven additionally samples form a Roman grid $(3 \times 3)$ replication experiment with $0.3 \mathrm{~m}$ equidistance.

The sampling rationale aimed at variographic fate characterization commensurate with a long profile at a scale length between 1 and $60 \mathrm{~m}$; the Roman square was intended as a basis for conventional statistical treatment (average and standard deviation). This central sample layout serves as a smallscale local "replication experiment" compared with the transect dimensions (Kardanpour et al., 2014). In total 64 sam- 
ples were collected: 57 samples from the long profile and 9 samples of the small grid, including 2 samples from the transect and the rest on the sides of the transect and between in a way to make a grid with 9 points. The original fresh soil was kept frozen until use.

The primary sampling was specifically intended to correspond to current sampling traditions in the soil and microbiology communities. In other studies efforts have been made to optimize each individual field sample, for example with respect to the famous "Gy's formula", from which control over the so-called fundamental sampling error is often sought. However, in the present study it is a major point to outline how the variographic approach, among other things, leads to a procedure with which to characterize the magnitude of the total sampling-plus-analytical error and thus to be warned of the need to control (better) all the inherent sampling errors; see, for example, DS3077 (2013) for a comprehensive introduction.

\subsection{Theory of sampling and variographic analysis}

The total analytical error (TAE) is most often under acceptable control in the analytical laboratory as regards both accuracy and precision. A sampling procedure must be both correct (ensures accuracy) and reproducible (ensures precision); TOS defines representativity in a rigid conceptual and mathematical approach. The critical issue is always, even for TOS-compliant sampling, that analytical results are but an estimate of the true (average) analytical grade of the lot sampled, because the aliquot is based on only a miniscule mass $(0.5-2.0 \mathrm{~g})$ compared to the entire field topsoil layer it is supposed to represent (typical mass / mass sampling ratios range from $1: 10^{3}$ to $\left.1: 10^{9}\right)$. The full sampling-analysis process and its characteristics are therefore the only guarantee for the relevance and reliability of the aliquot brought forth for analysis. The fundamental TOS principles need to be applied to all appropriate scales along the entire "fieldto-aliquot" pathway, not only to the primary sampling but in particular also to the successive stages of mass reduction in the laboratory before the ultimate analytical aliquot extraction. The only change in this multi-stage sampling chain is the operative scale (TOS principles and unit operations are scale-invariant). A comprehensive overview of all subsampling issues (laboratory mass reduction) can be found in Petersen et al. (2004), which does not include the "coningand-quartering" approach, despite the fact that this approach has enjoyed some popularity, e.g. for certain field applications to soils (Gerlach et al., 2002). However, the coningand-quartering approach has been severely criticized in the professional TOS literature, e.g. most recently in Esbensen and Wagner (2014); from a representativity point of view this mass reduction approach must be strongly discouraged.

On the basis of a correct sampling and mass reduction regimen, it is possible to characterize the inherent autocorrelation between units of a process/lot or along a 1-D transect (or transect). The semi-variogram (in this work referred to simply as the "variogram") is employed to describe the variation observed between sample pairs as a function of their internal distance.

To calculate a variogram, a sufficient number of units (increments/samples) are extracted equidistantly, spanning the process interval of interest, or the full transect length, as needed. The variogram is a function of a dimensionless, relative lag parameter, $j$, which is this distance between two units, the analytical results of which are compared. Full details of the variographic approach are described in DS3077 (2013), Esbensen et al. (2007, 2012a, b), Gy (1998), Minkkinen et al. (2012), and Petersen and Esbensen (2006), Petersen et al. (2005). Variograms may have apparent different specific appearances, but three fundamental characterizing features carry all the important information related to sampling errors and the heterogeneity along the transect in any-and-all variogram: the sill, the range, and the $y$ axis intercept, termed the nugget effect. Definitions of these features are given below.

The sill is the $y$ axis value at which the variogram levels off and becomes horizontal. The sill represents the total variance calculated from all experimental heterogeneity values. The sill corresponds to the overall maximum variance for the data series if/when calculated without taking their ordering into account.

The range is the lag distance beyond which the variogram $v(j)$ levels off and reaches a stable, constant sill. Samples taken at lags below the range are auto-correlated to a larger and larger degree as the lags become smaller and smaller. The range carries critical information as to the local heterogeneity with respect to the objective of the present method development.

The nugget effect indicates the amount by which the variance differs from zero when a variogram is extrapolated backwards so as to correspond to what would have been lag $=0$. A lag equal to zero has no physical meaning, but it represents the hypothetical case of two samples extracted at the same time and location (indeed from exactly the same physical volume of the lot). Thus, although "true replicates" from the exact same soil location (volume) are not physically possible, the nugget effect nevertheless allows for estimation of the corresponding discontinuous variance difference. This can be viewed as a collapse of the 1-D sampling situation (profile, transect) to a stationary sampling situation (small lots, 2-D and 3-D lots); see DS3077 (2013) and Esbensen et al. $(2007,2012 a, b)$ for further descriptions.

The nugget effect has a special interest; it contains all sampling, sample handling/processing, and analytical errors combined, which makes up the total measurement uncertainty. A variogram with a high nugget effect with regard to the sill signifies a measurement system not in sufficient control (DS3077, 2013; Esbensen and Wagner, 2014).

Variogram calculations are strongly influenced by outliers and/or trends. A valid variographic analysis often necessi- 


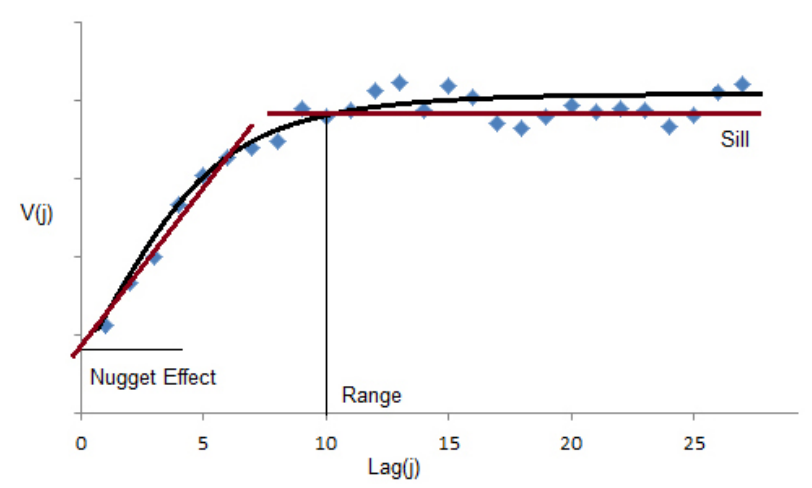

Figure 1. A generic variogram, schematically defining nugget effect, sill, and range. The illustration depicts an increasing variogram, which is the most often occurring type of variogram in the case of significant auto-correlation (for lags below the range) (Kardanpour et al., 2014). The nugget effect magnitude relative to the sill in this illustration is significant of an acceptable total measurement system, $<20 \%$.

tates outlier deletion after proper recognition and description and occasionally also de-trending of the raw transect data if/when trends are dominant or severe. In this study the raw data transect was de-trended using a simple regression slope subtraction from the data set where needed.

\subsection{Mass reduction/subsampling procedure}

After the stored samples were thawed and accommodated for $20{ }^{\circ} \mathrm{C}$ for a week, before being processed further, the primary field sample size (200-300 g) had to be reduced to the analytical sample size (1-2 g), not at all a trivial mass-handling issue. In order to provide representative subsamples, TOS principles were applied scrupulously to all mass reduction steps. Thus samples were dried and macerated, or ground, where appropriate, and subsequently deployed in a longitudinal tray, forming a 1-D lot, using the soil-adapted bedblending/cross-cut reclaiming technique described in detail in Petersen et al. (2004) and Kardanpour et al. (2015). These pre-blended micro-beds were cut by 10 randomly selected transverse increments along the elongated dimension which were aggregated, resulting in subsamples of 20-30 g each. The exact same procedure was repeated in a secondary mass reduction step further down, ending up with the final analytical mass $(2 \mathrm{~g})$ for the wet samples analyses. This procedure has been applied to provide full representativity in samples and to exclude all of the post-primary-sampling errors in order to be better able to focus on the latter and the variogram deployment (ibid).

The remainders of the secondary subsamples were airdried for 4 days at laboratory temperature $\left(20^{\circ} \mathrm{C}\right)$, to be used in parallel sorption experiments. As a further scale-down iteration, a similar bed-blending/cross-cut reclaiming was used to provide analytical samples of $2 \mathrm{~g}$, also based on 10 increments each.

Kardanpour et al. (2015) describe the "from-fieldsampling-to-aliquot" pathway in full detail, complete with an exhaustive pictorial presentation.

\subsection{Analytical experiment methods}

\subsubsection{MCPA sorption}

The sorption experiment started in glass vials with Teflon caps containing $1 \mathrm{~g}$ of the respective soils, and $9 \mathrm{~mL}$ of Milli$\mathrm{Q}$ water. The vials were kept for $24 \mathrm{~h}$ and then shaken in a horizontal, angled shaker prior to addition of $1 \mathrm{~mL}^{14} \mathrm{C}-$ MCPA stock solution, with $10000 \mathrm{dpm}$ in each individual vials. Sorption experiments were performed with two initial concentrations: 1 and $100 \mathrm{mg}$ of MCPA/L. Sorption was determined for MCPA in all of the 64 soil samples, using ${ }^{14} \mathrm{C}$ labelled MCPA.

After adding the stock solution, the vials were incubated in the shaker for $48 \mathrm{~h}$ and then placed vertically for another $48 \mathrm{~h}$, all at $20^{\circ} \mathrm{C}$. Subsequently $2 \mathrm{~mL}$ of the solution was transferred to the $2 \mathrm{~mL}$ Eppendorf micro-centrifuge tubes and centrifuged at $14500 \times g$ for $7 \mathrm{~min}$. Radioactivity in $1.5 \mathrm{~mL}$ of supernatant was determined using a Wallac 1409 liquid scintillation counter after mixing it with $10 \mathrm{~mL}$ of OptiPhase Hisafe3 scintillation cocktail.

\subsubsection{MCPA mineralization}

Mineralization experiments were carried out in a $100 \mathrm{~mL}$ glass jar with an airtight lid. Two-gram soil samples (wet weight) were placed in small plastic vials before adding $0.5 \mathrm{~mL}$ of ${ }^{14} \mathrm{C}$-labelled MCPA ( $5 \mathrm{mg}$ MCPA per gram of soil) with a radioactivity of $2000 \mathrm{dpm}$. A liquid scintillation counting (LSC) vial containing $2 \mathrm{~mL}$ of $0.2 \mathrm{M} \mathrm{NaOH}$ as a $\mathrm{CO}_{2}$ trap was also placed in the glass jar. The jars were incubated at $20^{\circ} \mathrm{C}$ for 14 days. Mineralization encountered as percentevolved ${ }^{14} \mathrm{CO}_{2}$ was measured at day 3,7 , and 14 . The $\mathrm{CO}_{2}$ traps were changed and replaced with a fresh trap at each sampling date. ${ }^{14} \mathrm{C}$ in the $\mathrm{NaOH}$ was measured as described in the sorption experiment by liquid scintillation counting.

\subsubsection{Biomass, substrate-induced respiration (SIR)}

The same setup as used for MCPA was used for the glucose mineralization, with adding $0.5 \mathrm{~mL}^{14} \mathrm{C}$-labelled glucose with $5000 \mathrm{dpm}$ to the $2 \mathrm{~g}$ of soil. All other setup, equipment, and experimental design details were identical. Alkaline traps were replaced with fresh alkaline traps and measured after 4 and $24 \mathrm{~h}$ considering the rapid respiration of the glucose and ${ }^{14} \mathrm{C}$ measured as described in the sorption experiment by liquid scintillation counting. Conversion into biomass was according to Dictor et al. (1998) and Tate et al. (1988). 


\subsubsection{Microbiology, bacteria colony formation units (CFUs)}

A suspension was made with $2 \mathrm{~g}$ of soil into $200 \mathrm{~mL}$ of sterile water, which was then shake for $15 \mathrm{~min}$ and diluted with sterilized water; this ended in two different dilutions for each sample, with differences of three and four orders of magnitude. To measure the soil microbiology, $1 \mathrm{~mL}$ of each sample was placed on a Petrifilm ${ }^{\circledR}$ (3M, Saint Paul, Minnesota, USA) sheet and CFUs were counted after 3 and 7 days of incubation at $20^{\circ} \mathrm{C}$.

\section{Results}

\subsection{Geochemical profiling}

In order to show the natural soil heterogeneity in a comparable format, Figs. 2-5 illustrate the individual large-scale parameter transects: concentration vs. location of the samples taken from the transect in the Fladerne field. Also shown is the variation in the central small-scale replication samples is shown as mean concentration $\pm 2 \mathrm{SD}$ with dashed horizontal lines in the figures. The large-scale variation in the soil moisture, LOI, and the biomass content are compared to the small-scale replication result for the same parameter in each graph (Fig. 2).

The same comparison graph (large scale/small scale) is illustrated for the MCPA sorption in Fig. 3 for two different initial MCPA concentrations, as it is clear; the soil sorption behaviour shows different variation with different concentrations. The results of the MCPA mineralization of the soil in Fig. 4 also show different variability in different mineralization steps. The transect of the MCPA mineralization is illustrated for different mineralization steps: the first 3 days, 4 to 7 days, and 8 to 14 days. The two latter periods show a rather similar variation because these two periods are in the final part of the mineralization development (Fig. 6).

The soil microbiology (log (CFU/g soil)) transect after 7 days of incubation is also illustrated in Fig. 5.

The Fladerne case represents an inherently very well mixed soil type, which has been managed by plowing for up to 100 years. The consequence of taking this lowheterogeneity end of the spectrum into account is that there is a limit to the degree of transect heterogeneity to be expected, as indeed witnessed in Figs. 2-5, where concentrations only comparatively rarely deviate outside the $\pm 2 \mathrm{SD}$ of the central Roman square design employed. However, this specific soil and tilling history feature must not lead to untoward confusion and illegitimate generalizations. It is the general applicability of the variographic approach which is illustrated here, as it happens, on a very well mixed substratum. Our parallel study showcases the approach on a significantly more heterogeneous case, in which the central Roman square does not bracket most of the transect concentration manifestations. This case was selected to represent the one
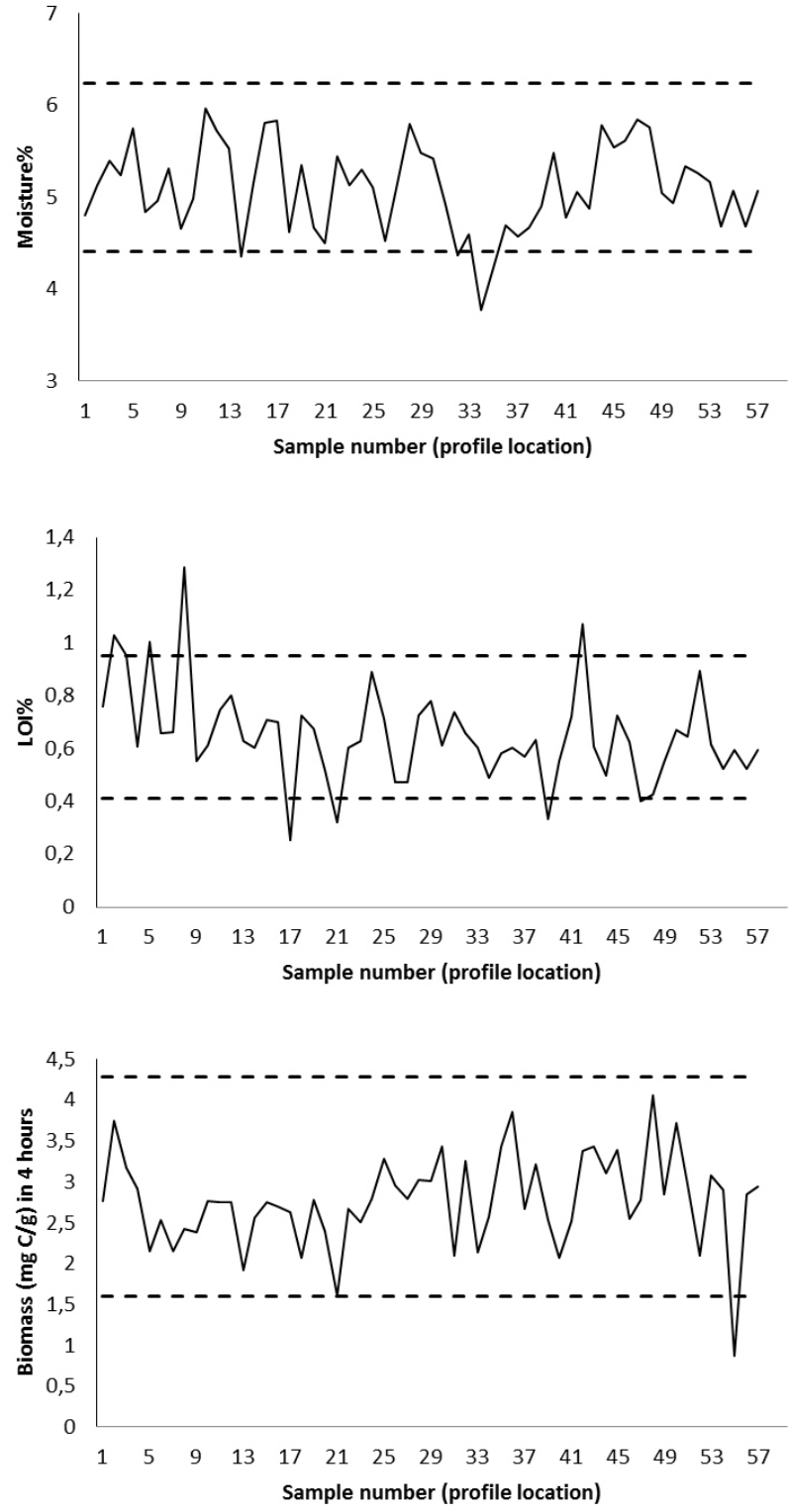

Figure 2. Fladerne Bæk. Transects of soil moisture (\%), LOI, and biomass $\left(\mathrm{mg} \mathrm{Cg}^{-1}\right)$ vs. soil sample number (transect location). Dashed lines represent mean $\pm 2 \mathrm{SD}$ of the small-scale replication experiment.

(almost extreme) end of a spectrum (only little inherent heterogeneity) from which to compare a whole spectrum of increasingly more heterogeneous soil types, horizons, and geological formations. Our own studies went a fair distance in this direction as possible with the Kardanpour et al. (2015), but obviously many even more heterogeneous cases exist and are to be found in the literature. 

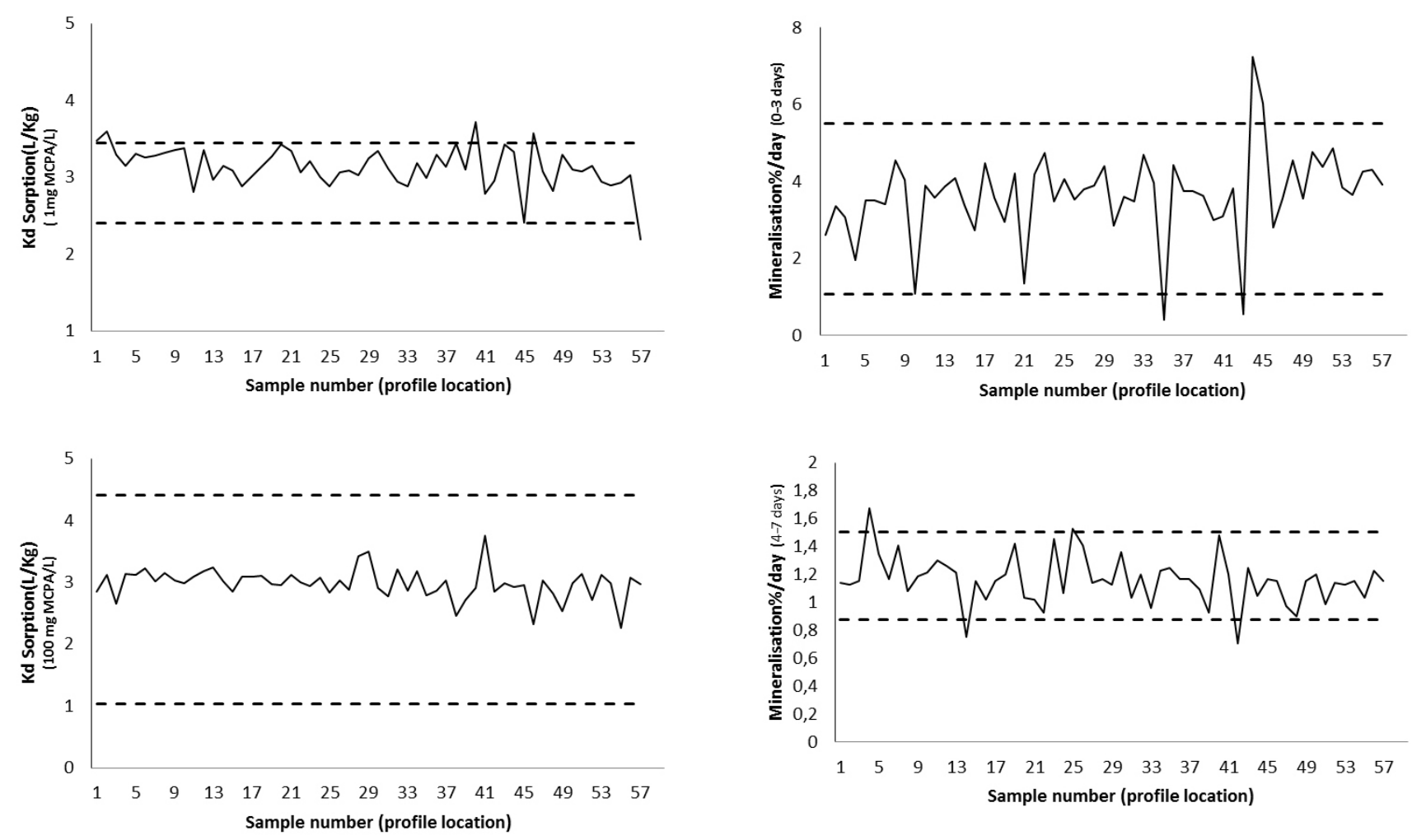

Figure 3. Fladerne Bæk. Transects of $K_{\mathrm{d}}$ MCPA sorption vs. sample number (transect location) $-K_{\mathrm{d}, 1}$ : MCPA $\left(1 \mathrm{mg} \mathrm{L}^{-1}\right) ; K_{\mathrm{d}, 100}$ : MCPA $\left(100 \mathrm{mg} \mathrm{L}^{-1}\right)$. Dashed lines represent mean $\pm 2 \mathrm{SD}$ of the small-scale replication experiment.

\subsection{Experimental variograms}

Prior to variogram calculation, all parameters were checked for outliers and trends (Figs. 2-5). Variograms were calculated using large-scale experimental transects without model fitting of the variogram parameters. This is common in geostatistics but not used here as TOS' variogram approach is not used for kriging but instead solely for heterogeneity characterization and interpretation.

Two different behaviours can be observed as displayed by two parameters groupings: the increasing Min1, LOI, and biomass variograms at the top, versus the remainder of parameters, which show a strongly similar form and behaviour (Fig. 7). As the sill levels represent the maximum parameter variation along the transect, parameters Min1, LOI, and biomass clearly display the highest transect variability. All variograms are of the increasing type with a distinct nugget effect. Following DS3077 (2013), the percentage nugget effect in relation to the sill, termed $\mathrm{RSV}_{1-\mathrm{dim}}$, is an expression of the total measurement uncertainty (MU) including total sampling error (TSE) (Esbensen and Wagner, 2014). In the present study this $\mathrm{MU}_{\text {total }}$ quality index ranges from $15 \%$ $\left(K_{\mathrm{d}, 100}\right)$ to $75 \%$ (Min1). There is thus an appreciable difference concerning the possibility to measure and character-

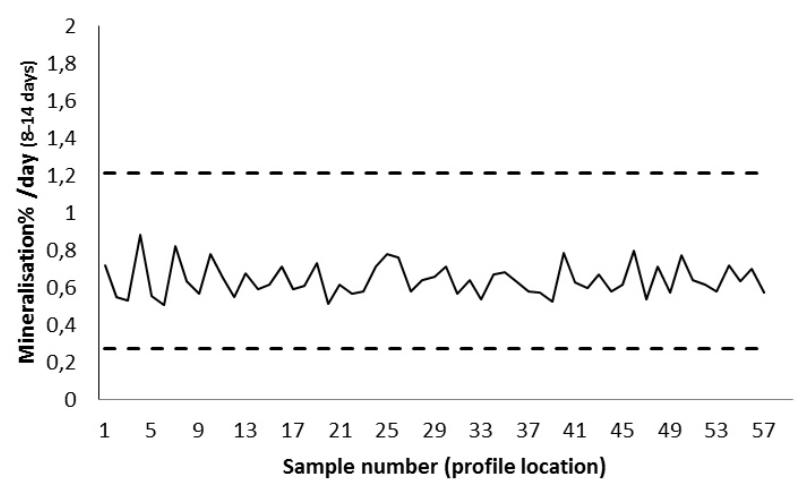

Figure 4. Fladerne Bæk. Transects of MCPA mineralization in three different periods, i.e. 0-3, 4-7, and 8-14 days, vs. sample number (transect location). Dashed lines represent mean $\pm 2 \mathrm{SD}$ of the small-scale replication experiment.

ize soil heterogeneity along the transect, ranging from very good to very poor. This facility for total measurement uncertainty validation is a powerful TOS benefit, with a wide carrying-over potential to many other sciences and fields of application. This feature is described in full in Esbensen and Romanach (2015) and Kardanpour et al. (2015), in which, incidentally, the 1-D transect of the present study appears in the form of a 1-D industrial process measurement series, illustrating the surprising generality of the variogram approach - modelling and interpretation and showing the way for application to natural processes in the geoscience and environmental science realms. 


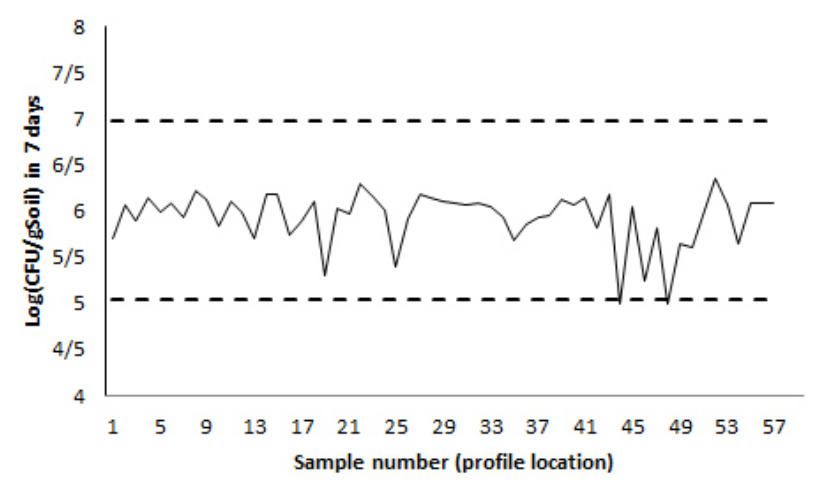

Figure 5. Fladerne Bæk. Transects of $\log (\mathrm{CFU} / \mathrm{g}$ soil) vs. sample number (transect location).

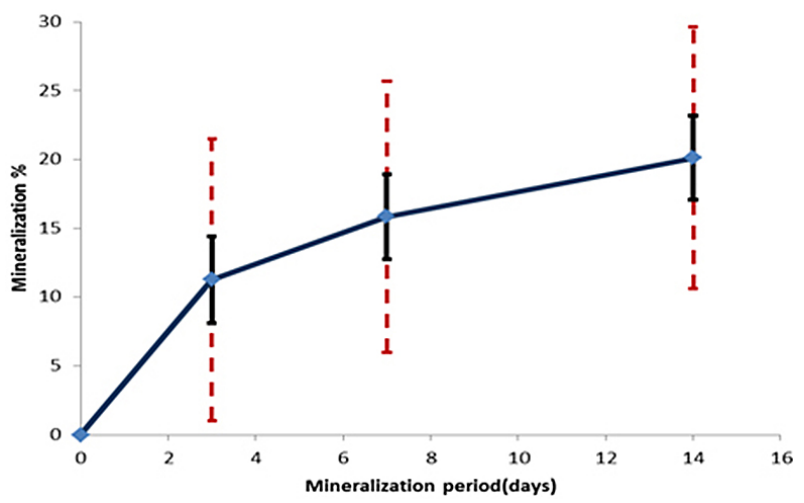

Figure 6. Average mineralization rate for all 57 samples: error bars are based on the standard deviation (solid bars) and the range of the whole sample set (dashed vertical bars).

Through application of the multivariate data analysis approach developed in the previous studies (Kardanpour et al., 2014, 2015), i.e. using the variograms as the input (X matrix) to a principal component analysis (PCA) with no centring and no scaling (see further below), the first component is found to represent $99 \%$ of the total variogram variance over all parameters, making it easy to find the average range characterizing the heterogeneity of the Fladerne transect, ca. $5 \mathrm{~m}$. Figure 8 shows the loadings for principal components 1 and 2, displayed in a fashion that mimics a spectrum. As expected, the PC-1 loadings delineate a general variogram shape, in fact presenting the average of all variograms in Fig. 7. The PC-2 loadings account for deviations from here, as caused by the individual variograms (mainly expressing a higher or lower average slope), a general feature that is markedly interprinted by random deviations. This component models the set of different slopes of the individual variograms, and it accounts for less than $1 \%$ total variance, but nevertheless it lends itself easily to interpretation as the wellknown spectroscopic "tilting" signature (Martens and Næs, 1991).

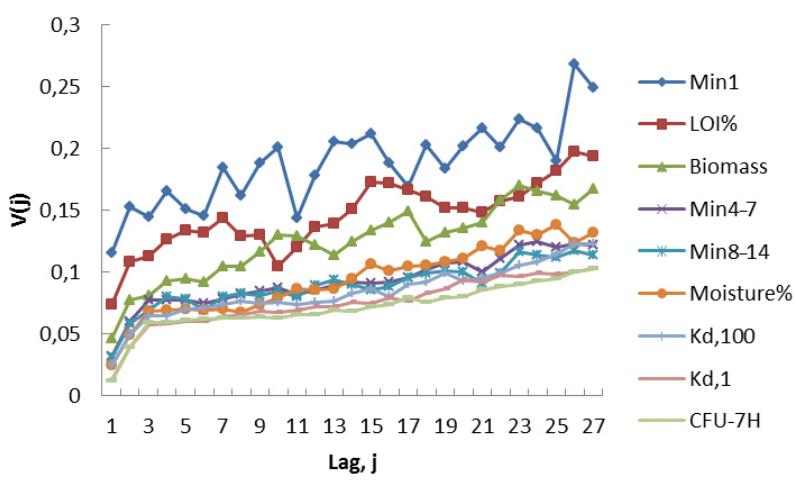

Figure 7. Synoptic variogram of all parameters in the present study comparing nugget effect, sill, and range levels.

In our earlier studies (e.g. Kardanpour et al., 2014), a pro et contra discussion can be found regarding pre-treatment of an X matrix made up of variograms. When basing variograms on heterogeneity contributions (a one-to-one transformation of the original analytical concentrations), this issue becomes moot, as this transformation is already performing what amounts to scaling. In the present paper we therefore did not apply centring, opting instead for the easily interpreted and useful appearance of the average variogram shape (Fig. 8, left).

\section{Discussion}

Aiming for a general approach to soil heterogeneity characterization, a set of naturally occurring organic, anthropogenic, and biota parameters were studied at scales from 1 to $60 \mathrm{~m}$ to be compared with other, for example minerogenic, parameters (see further below). The first step is always inspection of the raw data set with respect to potential outliers and/or trends. In the present study the geochemical parameter transects show no outliers and no strong trends (Figs. 2-5).

The experimental design allows comparison of the smallscale replication variability (classic statistics) and large-scale variability. All transects can, for example, be directly compared with the level and variation at the small-scale experiment (less than $1 \mathrm{~m}$ ), considering the pertinent mean $\pm 2 \mathrm{SD}$. In Figs. 2-5 the variation in the parameters in any selected small-scale window cannot be overestimated to the large scale; indeed, it also cannot be obtained from a small-scale replication study deviation estimate. This is just for visual orientation, however, and not to be confused with the nugget effect, a much more general characterization of the small(est) scale variability pertaining to below lag $=1$, summing up and averaging this information for all the sample pairs in the transect.

Any short interval on a transect (Figs. 2-5) can be considered as a small-scale study in its own right. In this context there is a clear difference between the empirical variability in different segments along each transect: the local 

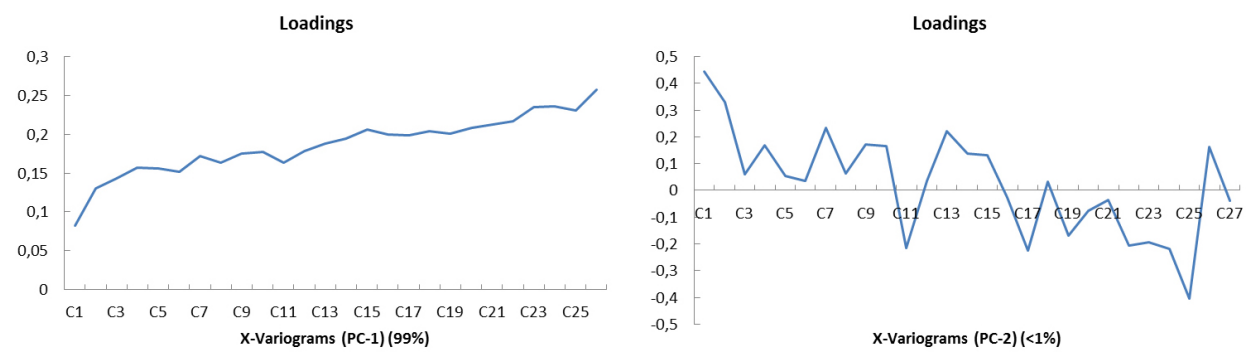

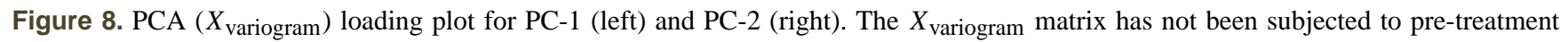
before PCA (no centring, no scaling). The range of the average variogram shape as represented by the PC-1 loadings is ca. $5 \mathrm{~m}$.

variability does not necessarily extend to larger scales. This has an important practical conclusion: no local small-scale sample collection can be generalized to larger scales. Unwitting or unreflected scaling-up of small-scale experimental organic, anthropogenic, and biota fate and mineralization results will bring an inflated uncertainty outside experimental control. The mineralization parameters which show different variation behaviour in the different mineralization steps send an important message regarding studies concerning time-dependent characterizations. A similar difference is observed for MCPA sorption with different concentrations, i.e. when studies are concerned with concentrationdependent phenomena.

The general local variability behaviour is, however, well captured as the below-range part of the general variogram loading spectrum for PC-1. The variogram is able to generalize the common local-scale behaviour. With TOS, there is synoptic information residing in the range, sill, and nugget effect for each individual parameter. Whenever heterogeneity variograms display a range, this relates to the ease and risk associated with attempting to secure field samples with minimum variability: sampling with smaller inter-increment lag distances than the range makes it possible to use the inherent auto-correlation between samples in a beneficial fashion.

From the earlier studies (Kardanpour et al., 2014, 2015) the overall conclusion was only to employ composite sampling. In the present context this means that, wherever practically possible, increments should only be collected with a maximum of half the observed range as a means to avoid unnecessary compositional variability effects due to the inherent soil heterogeneity. It follows that, in order to minimize the total sampling error, increments must be sampled with a maximum lag of $0.5 \times$ range, preferably smaller. In the present soil variograms a general range of $5 \mathrm{~m}$ is observed for multivariate variographic approach of the parameters (Fig. 8). It is evident that a thorough mixing of the selected set of increments is mandatory to sample locations with less than $2.5 \mathrm{~m}$ distance in between; for other soil types/analytes, other numerical magnitudes apply.

The variograms show different behaviour with respect to mineralization stages. This is expected from the slower rate of the mineralization in the latter stages (Fig. 6). The later stages display a flat variogram that only represents little autocorrelation between sample locations (Fig. 7) and the low sill level representing low variation along the transect. As is common in environmental studies, results of the mineralization are mostly reported in terms of the accumulated mineralization rate (see Fig. 6 as an example), i.e. results that are mostly affected by the first stages of the mineralization.

Most of the variograms level off quickly after only a few lags (range ca. $5 \mathrm{~m}$ ), followed by a flat (or slightly increasing) trend, whilst the first steps of MCPA mineralization, biomass, and LOI show more markedly increasing variograms (Fig. 7).

The CFU sill level is lower than natural organic and anthropogenic compounds, indicating lower variability in soil microbiology at the large scale(s). This can be compared with results from a series of other large-scale studies on different microbial communities for different anthropogenic and natural compound mineralization, which also showed that microbial biomass seems to be a stable intrinsic parameter of longer periods (Sørensen et al., 2003; Bending et al., 2001, 2003; Walker et al., 2001).

It is always a matter for discussion when theoretically anticipated correlations between the physiochemical/microbial activities fail to appear in specific real-world case studies. The more complex compounds have shown a more irregular, patchy fashion of decaying due to more specific microbial communities (but still generally isotropic in nature). Analysis of soil parameters rarely gives a clear pattern; this seems to be associated with a number of non-included or unknown parameters, resulting in a high degradation potential in some cases but low elsewhere (Sørensen et al., 2003; Rasmussen et al., 2005; Bending et al., 2001; Walker et al., 2001). Upon reflection, however, this is no mystery but simply a result of local soil heterogeneity, which cannot be formulated or predicted based on the physiochemical biological or microbial correlation of the properties of soil in large-scale studies. A variographic heterogeneity characterization at all scales is thus a beneficial pilot experiment able to focus on the relevant heterogeneities characterizing individual, or groups of, parameters in their proper scale-dependent relationships.

To sum up the results of all measured parameters studied here, for environmental purposes and objectives related to soil parameters at field scale, it is advantageous to employ a 
variographic heterogeneity characterization as a pilot study. Results here will lead to a comprehensive understanding of the spatial variability and auto-correlation of the parameters in the field.

The results from the present study show that, for wellmixed sandy soil, it is recommended to sample locations with less than $2.5 \mathrm{~m}$ inter-distance in between, preferably smaller. It is necessary to conduct a similar variographic pilot experiment in order to outline the relevant scale-heterogeneity characteristics for other soil types, which, unavoidably, will tend to show more irregular spatial heterogeneity patterns - each principal soil type will in principle be characterized by a specific range, but there is a further caveat. Each analyte may in fact display its own more or less specific range, as witnessed above, as well as in a large number of studies in the literature. When controlling the spatial heterogeneity is of the essence, the logical solution is to design the sampling according to the analyte with the smallest range, i.e. the most heterogeneously distributed analyte - this will by necessity also take care of all other analytes with higher ranges. If the emphasis is on sampling costs, a not completely unlikely alternative scenario that may or may not clash with representativity, it is a comforting thought that all analytes are measured on the same final aliquot. By carefully optimizing the primary field sampling according to the principles presented here, all analytes will be measured with the same, optimal relevance, indeed with regard to the same representativity. If sampling is done right from the start, there are no extra costs - the opposite, however, is a very different case, as should be abundantly clear.

Results from a parallel study on the minerogenic compounds for the same Fladerne field (Kardanpour et al., 2014) show a similar soil heterogeneity compared to the present anthropogenic compounds. The nugget effects for most of the minerogenic compounds are of the same order of magnitude as those for the anthropogenic compounds - i.e. the total measurement system and procedures (sampling/handling/processing/analysis) pass all the quality criteria for representative sampling established in the recent sampling standard (DS3077, 2013).

In cases where the next step in studies might be assessment of the main factors driving the spatial heterogeneity of soil contamination analytes, for example, the 1-D (or 2$\mathrm{D} X-Y$ ) approach advocated here will only serve as a basis for proper selection of experimental material to be taken to the laboratory - upon which further considerations will focus on, say, the potential factors involved in contaminant input and transport, among other things. Note that these latter processes manifest themselves primarily in the $Z$ direction, where it is by no means a given that application of the same variographic approach (or geostatistical modelling) will necessary give meaningful results.

\section{Conclusions}

A pilot experiment aimed at an intrinsic 1-D soil heterogeneity characterization is a critical success factor for laboratory studies relying on field samples to provide the experimental pots, which for replicate and comparative study objectives need to be as similar as at all possible. As a case study, the variographic results for sandy soils show that the distance between two sample spot must be less than $2.5 \mathrm{~m}$ for the present set of organic compounds and soil type. Specific soil types and/or other analytes will in principle display different ranges and nugget effects, and hence our call for systematic deployment of the variographic pilot experiment, from which all necessary information can be derived for designing an optimal sampling plan, e.g. identifying the analyte with the smallest range (for significantly correlated analytes). For the case of well-mixed soil components, a general PCA approach for modelling a whole set of variograms may be useful in addition to individual analyte consideration.

Without these types of information, experimental fate study work is essentially devoid of a valid basis as regards interpretation, scaling up, and scientific generalization of the experimental results back to the field scale. A large-scale 1$\mathrm{D}$ transect sampling can reveal the inherent heterogeneity at all scales, from the smallest local sampling equidistance up to the maximum experimental length scale studied. Variographic analysis was here employed successfully to soil heterogeneity at scales between 1 and $100 \mathrm{~m}$; other scenarios may require other numerical parameters, while the general approach remains identical.

The TOS-guided variogram pilot study approach illustrated here has a substantial carrying-over potential to geochemistry and environmental science, as well as other areas of application. It is even applicable to dynamic systems, i.e. to natural or technological processes in these realms.

Acknowledgements. The authors gratefully acknowledge the Danish Research Council for $\mathrm{PhD}$ stipend funding (stipend no. 562/06-18-10028(6)) to Z. Kardanpour and valuable laboratory services and assistance provided by GEUS, Dept. of Geochemistry. The authors are grateful to GEUS' management for a positive attitude with respect to development and application of TOS' principles of proper representative sampling, which are not always recognized in geoscience communities.

Edited by: O. Evrard 


\section{References}

Adamchuk, V. I., Viscarra Rossel, R. A., Marx, D. B., and Samal, A. K.: Using Targeted Sampling to Process Multivariate Soil Sensing Data, Geoderma, 163, 63-73, 2011.

Arias-Estévez, M., López-Periago, E., Martínez-Carballo, E., Simal-Gándara, J., Mejuto, J.-C., and García-Río, L.: The Mobility and Degradation of Pesticides in Soils and the Pollution of Groundwater Resources, Agr. Ecosyst. Environ., 123, 247-260, 2008.

Bending, G., Shaw, E., and Walker, A.: Spatial Heterogeneity in the Metabolism and Dynamics of Isoproturon Degrading Microbial Communities in Soil, Biol. Fert. Soils, 33, 484-489, 2001.

Bending, G. D., Lincoln, S. D., Sebastian, R., Morgan, J. A. W., Aamand, J., Sørensen, S. R., and Walker, A.: In-Field Spatial Variability in the Degradation of the Phenyl-Urea Herbicide Isoproturon Is the Result of Interactions between Degradative Sphingomonas Spp. and Soil pH, App. Environ. Microbiol., 69, 827834, 2003.

Boudreault, J.-P., Dubé, J.-S., Sona, M., and Hardy, E.: Analysis of Procedures for Sampling Contaminated Soil Using Gy's Sampling Theory and Practice, Sci. Total Environ., 425, 199-207, 2012.

Chappell, A. and Viscarra Rossel, R. A.: The Importance of Sampling Support for Explaining Change in Soil Organic Carbon, Geoderma, 193-194, 323-325, 2013.

Crespin, M. A., Gallego, M., Valcárcel, M., and González, J. L.: Study of the Degradation of the Herbicides 2,4-D and MCPA at Different Depths in Contaminated Agricultural Soil, Environ. Sci. Technol., 35, 4265-4270, 2001.

De Zorzi, P., Barbizzi, S., Belli, M., Fajgelj, A., Jacimovic, R., Jeran, Z., Sansone, U., and van der Perk, M.: A Soil Sampling Reference Site: The Challenge in Defining Reference Material for Sampling, Appl. Radiat. Isotopes, 66, 1588-1591, 2008.

Dictor, M.-C., Tessier, L., and Soulas, G.: Reassessment of theK EC Coefficient of the Fumigation \pm Extraction Method in a Soil Profile, Soil Biol. Biochem., 30, 119-127, 1998.

DS3077: Representative Sampling/ Horizontal Standard, Danish Standard Authority, 44, 1-38, 2013.

Esbensen, K. H. and Romanach, R. J.: Counteracting soil heterogeneity sampling for environmental studies (pesticide residues, contaminants transformation) - TOS is critical, Proceedings 7th World Conference on Sampling and Blending (WCSB7), 205209, 2015.

Esbensen, K. H. and Wagner, C.: Theory of Sampling (TOS) versus Measurement Uncertainty (MU) - A Call for Integration, TrACTrend. Anal. Chem., 57, 93-106, 2014.

Esbensen, K. H., Friis-Petersen, H. H., Petersen, L., Holm-Nielsen, J. B., and Mortensen, P. P.: Representative Process Sampling in Practice: Variographic Analysis and Estimation of Total Sampling Errors (TSE), Chemometr. Intell. Lab., 88, 41-59, 2007.

Esbensen, K. H., Paoletti, C., and Minkkinen, P.: Representative Sampling of Large Kernel Lots I. Theory of Sampling and Variographic Analysis, TrAC-Trend. Anal. Chem., 32, 154-164, 2012a.

Esbensen, K. H., Paoletti, C., and Minkkinen, P.: Representative Sampling of Large Kernel Lots III. General Considerations on Sampling Heterogeneous Foods, TrAC-Trend. Anal. Chem., 32, 178-184, 2012b.
Gerlach, R. W., Dobb, D. E., Raab, G. A., and Nocerino, J. M.: Gy Sampling Theory in Environmental Studies. 1. Assessing Soil Splitting Protocols, J. Chemometr., 16, 321-328, 2002.

Gy, P. M.: Sampling for Analytical Purposes, 1st Edn. Chichester, West Sussex, UK, John Wily \& Sons, 172 pp., ISBN: 978-0-47197956-2, 1998.

Johnsen, A. R., Styrishave, B., and Aamand, J.: Quantification of Small-Scale Variation in the Size and Composition of Phenanthrene-Degrader Populations and PAH Contaminants in Traffic-Impacted Topsoil, FEMS Microbiol. Ecol., 88, 84-93, 2014.

Kardanpour, Z., Jacobsen, O. S., and Esbensen, K. H.: Soil Heterogeneity Characterization Using PCA (Xvariogram) - Multivariate Analysis of Spatial Signatures for Optimal Sampling Purposes, Chemometr. Intell. Lab., 136, 24-35, 2014.

Kardanpour, Z., Jacobsen, O. S., and Esbensen, K. H.: Counteracting soil heterogeneity sampling for environmental studies (pesticide residues, contaminants transformation) - TOS is critical, Proceedings 7th World Conference on Sampling and Blending (WCSB7), 205-209, 2015.

Li, B. G., Cao, J., Liu, W. X., Shen, W. R., Wang, X. J., and Tao, S.: Geostatistical Analysis and Kriging of Hexachlorocyclohexane Residues in Topsoil from Tianjin, China, Environ. Pollut., 142, 567-575, 2006.

Lin, Q., Li, H., Luo, W., Lin, Z., and Li, B.: Optimal Soil-Sampling Design for Rubber Tree Management Based on Fuzzy Clustering, Forest Ecol. Manage., 308, 214-222, 2013.

Martens, H. and Næs, T.: Multivariate Calibration, John Wiley \& Sons, Chichester, West Sussex, UK, 438 pp., ISBN: 978-0-47193047-1, 1991.

Minkkinen, P., Esbensen, K. H., and Paoletti, C.: Representative Sampling of Large Kernel Lots II. Application to Soybean Sampling for GMO Control, TrAC-Trend. Anal. Chem., 32, 165-177, 2012.

Mulder, V. L., de Bruin, S., and Schaepman, M. E.: Representing Major Soil Variability at Regional Scale by Constrained Latin Hypercube Sampling of Remote Sensing Data, Int. J. Appl. Earth Obs., 21, 301-310, 2013.

Petersen, L. and Esbensen, K. H.: Representative Process Sampling for Reliable Data Analysis - a Tutorial, J. Chemometr., 19, 625647, 2006.

Petersen, L., Dahl, C. K., and Esbensen, K. H.: Representative Mass Reduction in Sampling - a Critical Survey of Techniques and Hardware, Chemometr. Intell. Lab., 74, 95-114, 2004.

Petersen, L., Minkkinen, P., and Esbensen, K. H.: Representative Sampling for Reliable Data Analysis: Theory of Sampling, Chemometr. Intell. Lab., 77, 261-277, 2005.

Rasmussen, J., Aamand, J., Rosenberg, P., Jacobsen, O. S., and Sørensen, S. R.: Spatial Variability in the Mineralisation of the Phenylurea Herbicide Linuron within a Danish Agricultural Field: Multivariate Correlation to Simple Soil Parameters, Pest Manage. Sci., 61, 829-837, 2005.

Rodriguez-Cruz, M. S., Jones, J. E., and Bending, G. D.: FieldScale Study of the Variability in Pesticide Biodegradation with Soil Depth and Its Relationship with Soil Characteristics, Soil Biol. Biochem., 38, 2910-2918, 2006.

Rosenbom, A. E., Binning, P. J., Aamand, J., Dechesne, A., Smets, B. F., and Johnsen, A. R.: Does Microbial Centimeter-Scale Het- 
erogeneity Impact MCPA Degradation in and Leaching from a Loamy Agricultural Soil?, Sci. Total Environ., 472, 90-98, 2014. Sørensen, S. R., Bending, G. D., Jacobsen, C. S., Walker, A., and Aamand, J.: Microbial Degradation of Isoproturon and Related Phenylurea Herbicides in and below Agricultural Fields, FEMS Microbiol. Ecol., 45, 1-11, 2003.

Sørensen, S. R., Schultz, A., Jacobsen, O. S., and Aamand, J.: Sorption, Desorption and Mineralisation of the Herbicides Glyphosate and MCPA in Samples from Two Danish Soil and Subsurface Profiles, Environ. Pollut., 141, 184-194, 2006.

Tate, K. R., Ross, D. J., and Feltham, C. W.: A Direct Extraction Method to Estimate Soil Microbiology C: Effects of Experimental Variables and Some Different Calibration Procedures, Soil Biol. Biochem., 20, 329-335, 1988.
Torstensson, N. T. L. and Stark, J.: The Effect of Repeated Applications of 2, 4-D and MCPA on Their Breakdown in Soil, Weed Res., 15, 159-164, 1975.

Totaro, S., Coratza, P., Durante, C., Foca, G., Li Vigni, M., Marchetti, A., Marchetti, M., and Cocchi, M.: Soil Sampling Planning in Traceability Studies by Means of Experimental Design Approaches, Chemometr. Intell. Lab., 124, 14-20, 2013.

Walker, A., Jurado-Exposito, M., Bending, G. D., and Smith, V. J.: Spatial Variability in the Degradation Rate of Isoproturon in Soil, Environ. Pollut., 111, 407-415, 2001. 